\title{
Film thickness in elastohydrodynamically lubricated elliptic contacts
}

\author{
G. Nijenbanning, C.H. Venner, H. Moes \\ University of Twente, Faculty of Mechanical Engineering, P.O. Box 217, 7500 AE Enschede, Netherlands
}

Received 7 January 1994; accepted 31 March 1994

\begin{abstract}
A multilevel solver for the circular contact was extended to elliptical contact problems. After verification of its predictions by comparison with results presented in literature, it was used to study the variations of film thickness with varying operating conditions and aspect ratio of the contact ellipse. Detailed computational results are presented and observed tendencies are traced back to the modelling equations. Subsequently it is demonstrated how and when, for contacts with the entrainment directed perpendicular to the major principal axis of the contact ellipse, the pressure and film thickness on the centre-line of the contact can be predicted accurately from an equivalent line contact analysis. Finally, survey graphs of the minimum and the central film thickness are presented and a formula is given that predicts the central film thickness as a function of load and lubricant parameters, and the ratio of reduced radii of curvature of the surfaces. This formula incorporates asymptotic behaviour and as a result it can be applied for all conditions. In particular, its accuracy for contacts with the major principal axis of the contact ellipse perpendicular to the entraining direction is demonstrated in this paper.
\end{abstract}

Keywords: Elliptic contacts; Contacts; Film thickness; Elastohydrodynamic; Lubrication

\section{Introduction}

Lubricated contacts in technical equipment and machines often operate in the regime referred to as elastohydrodynamic lubrication (EHL), e.g. the contacts between gear teeth, between the rolling element and the inner or outer raceway in rolling element bearings, between an elastomeric seal and a rod or between a cam and follower.

This type of lubrication has been studied intensively over the past decades, both theoretically and experimentally. Since the introduction of the digital computer, theoretical studies often involve the numerical solution of the pressure profile in the contact and the shape of the lubricant film from a set of differential and integral equations describing the lubricant flow, the lubricant behaviour and the surface deformation. In the early years this research was quite often hampered by computing time problems and poor stability behaviour of the numerical algorithms, particularly for the high loads occurring in practical contacts between steel surfaces. However, as a result of developments on the software side, more efficient and stable algorithms, aided by developments on the hardware side, faster computers and workstations, increasingly complex simulations can be performed nowadays. Together with the experimental investigations, these theoretical studies have contributed to the current quite high level of understanding of the physical mechanisms determining operation and service life of EHL contacts. However, many questions are still to be answered, for example, with respect to elliptical contacts.

Hamrock and Dowson [1] were among the first to present numerical results for these contacts. From their results they derived a formula to predict the film thickness as a function of the operating conditions. Using a similar numerical algorithm, Chittenden et al. [2] extended this work and presented an improved film thickness formula. However, as the formula does not include asymptotic behaviour, it is of relatively limited applicability.

More recent are the numerical results presented by Seabra and Berthe [3,4], Kweh et al. [5], Barragan de Ling [6] and Evans and Snidle [7]. These studies, however, place emphasis on specific aspects, i.e. on surface features or on a particular gear design, and 
results are presented for a limited range of conditions only. Alternatively, studies with the aim to obtain a survey of the film thickness behaviour as a function of load parameters, lubricant parameters and the geometry of the contact were carried out, for example, by Chittenden et al. [8] (elastic isoviscous regime) and by Hooke [9]. Assuming relatively soft contacts, Hooke considered different regions of the contact separately, i.e. the central region and the region of the so-called side-lobes. From these analyses, equations for the minimum film thickness were derived. Later, charts for determining the minimum film thickness were presented by Hooke [10].

In this paper the elliptic contact problem is revisited. The multilevel algorithm for the numerical solution of the circular contact problem introduced by Venner [11] was extended to such contacts. For verification, two loading cases considered by Kweh et al. [5] were taken and the results were compared. Subsequently, the algorithm was used to investigate the variations in the film thickness with varying operating conditions and curvature ratio in contacts with the entrainment directed perpendicular to the major principal axis of the contact ellipse, i.e. so-called wide elliptical contacts. A selection of the numerical results will be presented and some tendencies observed are traced back to the basic equations. Special attention is given to the asymptotic behaviour with increasing width of the contact ellipse, i.e. it is investigated how accurately pressure profile and film thickness on the centre-line of the contact can be predicted from a (more simple) line contact analysis.

Finally, survey charts of both the minimum and the central film thickness are presented and a formula is derived from the results, which predicts the central film thickness as a function of the operating conditions and curvature ratio. An advantage of this formula over existing formulas is that it incorporates asymptotic behaviour and thereby has the prospect of validity over the widest possible range of conditions. In this paper its accuracy for the aforementioned class of elliptical contacts is demonstrated.

\section{Theory}

Below, the physical-mathematical model for an elliptical contact that has been applied will be introduced, followed by a brief discussion dealing with the dimensionless parameters that can bc used to characterise load situations. The model is based upon Reynolds' equation [12] and the theory of deformation of elastic half-spaces, see Love [13]. The lubricant is characterised by its (pressure-dependent) viscosity and density. Hence, its behaviour is assumed to be Newtonian, an assumption that is justified because in the present study emphasis is on the pressure and the film thickness and not on the prediction of friction.

\subsection{Equations}

Defining the following dimensionless variables:

$X=x / a_{x} \quad Y=y / a_{x}$

$P=p / p_{h} \quad H=h R_{x} / a_{x}^{2}$

$\bar{\eta}=\eta / \eta_{0} \quad \bar{\rho}=\rho / \rho_{0}$

Reynolds' equation for a steady state, isothermal point contact with lubricant entrainment along the $x$ direction can be written as:

$\frac{\partial}{\partial X}\left(\epsilon \frac{\partial P}{\partial X}\right)+\frac{\partial}{\partial Y}\left(\epsilon \frac{\partial P}{\partial Y}\right)-\frac{\partial(\bar{\rho} H)}{\partial X}=0$

with the cavitation condition $P \geqslant 0$ throughout the domain and the boundary conditions $P\left(X_{\mathrm{a}}, Y\right)=P\left(X_{\mathrm{b}}, Y\right)=0$ and $P\left(X,-Y_{\mathrm{a}}\right)=P\left(X, Y_{\mathrm{a}}\right)=0$. The coefficient $\epsilon$ is defined by:

$\epsilon=\frac{\bar{\rho} H^{3}}{\bar{\eta} \lambda}$

where $\lambda$ denotes a dimensionless speed parameter:

$\lambda=\frac{6 u_{\mathrm{s}} \eta_{0} R_{x}^{2}}{a_{x}^{3} p_{\mathrm{h}}}$

For the viscosity-pressure relation, the Barus equation [14] and the Roelands equation [15] will be used. The lubricant compressibility is modelled with the Dowson and Higginson equation [16].

In terms of the dimensionless variables the film thickness equation reads:

$$
\begin{aligned}
H(X, Y)= & H_{0}+\frac{X^{2}}{2}+D \frac{Y^{2}}{2} \\
& +\frac{1+D}{2 \pi E_{\mathrm{c}}} \int_{\Omega} \frac{P\left(X^{\prime}, Y^{\prime}\right) \mathrm{d} X^{\prime} \mathrm{d} Y^{\prime}}{\sqrt{\left(X-X^{\prime}\right)^{2}+\left(Y-Y^{\prime}\right)^{2}}}
\end{aligned}
$$

In this equation $D=R_{x} / R_{y}$ denotes the ratio of the reduced radii of curvature of the contacting surfaces (see Appendix A), $E_{\mathrm{c}}$ the Legendre normal, or complete elliptic integral of the second kind as used in the Hertzian dry contact theory [17] (sce Appendix B), and $H_{0}$ an integration constant.

The solution is subject to the following global condition of force balance:

$$
\int_{-\infty}^{\infty} \int_{-\infty}^{\infty} P(X, Y) \mathrm{d} X \mathrm{~d} Y-\frac{2 \pi}{3 \kappa}=0
$$

where $\kappa=a_{x} / a_{y}$ denotes the aspect ratio of the contact ellipse. $\kappa$ can be computed once $D$ is known; see Appendix B. 


\subsection{Parameters}

For the physical-mathematical model as presented above, the number of dimensionless groups needed to characterise the contact fully depends on the viscosity-pressure and density-pressure relations used. If the lubricant is assumed to be incompressible and Barus' equation is used, three dimensionless parameters are sufficient, i.e. $D$ and $\lambda$, as given above, and $\bar{\alpha}=\alpha p_{\mathrm{h}}$. Instead of $\bar{\alpha}$ and $\lambda$, alternatively the dimensionless parameters $M$ and $L$ introduced by Moes [18] can be used:

$M=\frac{F}{E^{\prime} R_{r}^{2}}\left(\frac{\eta_{0} u_{\mathrm{s}}}{E^{\prime} R_{x}}\right)^{-3 / 4}$

$L=\alpha E^{\prime}\left(\frac{\eta_{0} u_{\mathrm{s}}}{E^{\prime} R_{x}}\right)^{1 / 4}$

In this paper the latter parameters (in addition to $D$ ) will be used for characterising the load situations. The relation between $M, L$ and $\bar{\alpha}, \lambda$ can be found in Appendix C. If Barus' equation is replaced by Roelands' equation and compressibility (e.g. according to Dowson and Higginson) is taken into account, two additional parameters should be introduced. However, if in that case the pressure-viscosity coefficient $\alpha$ and the pressure-viscosity index $z$ are assumed to be given, once again three parameters suffice $(M, L$, and $D)$. This approach has been followed here and, unless explicitly stated otherwise (see section 4), results obtained using Roelands' equation and Dowson and Higginson's equation apply to $\alpha=2.2 \times 10^{-8} \mathrm{~Pa}^{-1}$ and $z=0.68$.

\section{Numerical solution}

The equations to be solved, i.e. Eqs. (1), (2) and (3), are not essentially different from the equations describing the circular contact problem $(D=1)$. Obviously, they can be discretised in the same way but, more importantly, the principal terms determining how to solve the problem numerically, remain the same. Consequently, the analysis as presented by Venner [11] also applies here, and with some minor modifications the same numerical solution algorithm can be used. For details with respect to this algorithm the reader is referred to Refs. [11] and [19]; however, for completeness a brief general description is given below. The equations are discretised on a uniform grid and the resulting system of equations is solved using multigrid techniques. An introduction to these techniques is given by Briggs [20]. Applications to a variety of problems can be found in Ref. [21], whereas the implementation for EHL problems is discussed in detail by Venner [11] and by Lubrecht [22].
The core of the algorithm is a relaxation scheme that, by exploiting the local behaviour of the problem, i.e. its integral character in regions of small pressure flow and its differential character in regions of dominating pressure flow, is stable for a wide range of loading conditions. This relaxation is embedded in a full multigrid algorithm (FMG), where the so-called full approximation scheme (FAS) is used to deal with the non-linearity, see Ref. [21]. In such an FMG algorithm, in addition to the grid on which the solution is desired, a set of coarser grids is used for two purposes. Firstly, they are used to generate an accurate first approximation on the finest grid. Secondly, in a process referred to as the coarse grid correction cycle, they serve to obtain a speed of convergence that is independent of mesh size. Because of the multi-summations resulting from the discretisation of the elastic deformation integrals, the above procedure is not yet sufficient to obtain optimal efficiency. The final step to achieve that goal is the use of the coarser grids to fulfil an additional task, i.e. multilevel multi-integration, a multigrid technique for the fast evaluation of these summations; see Ref. [23]. The resulting algorithm enables solution of the problem in $O(n \ln n)$ operations if $n$ is the number of nodes on the grid. Consequently, even on small capacity computers, dense grids may be used and extensive parameter studies may be carried out. For the circular contact this is shown in detail by Venner [11] and by Venner and ten Napel [19,24].

\section{Verification}

Using a mixed inverse-direct numerical solution algorithm on a single grid, Kweh et al. [5] presented solutions for elliptical contacts with surface disturbances. The pressure profile and the film thickness were computed using the Barus viscosity-pressure equation, and the Dowson and Higginson density-pressure equation. From their paper two contact situations were taken as test cases to verify the algorithm developed. For completeness the values of the different parameters describing these two cases and the resulting values of the various dimensionless parameters are listed in Table 1.

For the present study both cases were solved using a domain extending from -2.5 to 1.5 in the $X$ direction and from -8 to 8 in the $Y$ direction. The finest grid (highest level) used in the FMG algorithm consisted of $257 \times 1025$ points. Table 2 lists the values for the minimum and central film thickness obtained on the different grids, together with the values presented for these cases by Kweh et al. [5]. Each time the grid is refined, the mesh size halves. This enables verification of the accuracy of the discretisation, i.e. of the convergence of the numerical solution to the continuous 
Table 1

Load conditions as used by Kweh et al. [19, Table 1]

\begin{tabular}{|c|c|c|c|}
\hline Parameter & \multicolumn{2}{|c|}{ Value } & Unit \\
\hline$R_{x}$ & \multicolumn{2}{|c|}{0.01905} & $\mathrm{~m}$ \\
\hline$F$ & \multicolumn{2}{|c|}{992.4} & $\mathrm{~N}$ \\
\hline$u_{\mathrm{s}}$ & \multicolumn{2}{|c|}{49.74} & $\mathrm{~ms}^{-1}$ \\
\hline$E_{1}-E_{2}$ & \multicolumn{2}{|c|}{$2.06810^{11}$} & $\mathrm{~N} \mathrm{~m}^{2}$ \\
\hline$\nu_{1}=\nu_{2}$ & \multicolumn{2}{|c|}{0.3} & \\
\hline$\eta_{0}$ & $17.910^{-3}$ & $2.410^{-3}$ & Pa s \\
\hline$\alpha$ & $1.4810^{-8}$ & $0.9610^{-8}$ & $\mathrm{~Pa}^{-\mathrm{I}}$ \\
\hline$D$ & 0.125 & 0.125 & \\
\hline$M$ & 221.56 & 1000 & \\
\hline$L$ & 12.74 & 5 & \\
\hline $\bar{\alpha}$ & 15.686 & 10.174 & \\
\hline$\lambda$ & 0.0470 & 0.0063 & \\
\hline
\end{tabular}

Table 2

Minimum and central film thickness in micrometres obtained in grids with decreasing mesh size, and values presented by Kweh et al. [19, Table 3].

\begin{tabular}{llllll}
\hline \multirow{2}{*}{ Level } & \multicolumn{2}{l}{ Case A } & & & \multicolumn{2}{l}{ Case B } \\
\cline { 2 - 3 } \cline { 5 - 6 } & $h_{\min }$ & $h_{\text {cen }}$ & & $h_{\text {min }}$ & $h_{\text {cen }}$ \\
\hline 5 & 1.104 & 1.364 & 0.194 & 0.276 \\
6 & 1.193 & 1.401 & 0.233 & 0.270 \\
7 & 1.246 & 1.424 & 0.243 & 0.282 \\
8 & 1.257 & 1.430 & 0.246 & 0.285 \\
Kweh et al. & 1.256 & 1.341 & 0.249 & 0.283 \\
\hline
\end{tabular}

solution of the problem. Table 2 shows that the values of minimum and central film thickness converge in a first-order manner to a limiting value. This is in accordance with the fact that the algorithm is based upon a first-order discretisation. From Table 2 it can be concluded that the accuracy of the final solution (level 8 ) is about $1 \%$.

Notice the close agreement between the values obtained with the algorithm developed and those presented by Kweh et al. for these loading cases.

\section{Results}

Figs. 1 and 2 show the calculated dimensionless pressure profile $P$ and the associated dimensionless film thickness $H$ for $M=1000, L=10$ and $D=0.4$. Similarly, Figs. 3 and 4 show $P$ and $H$ for the same $M$ and $L$ but with $D=0.2$. The solutions were obtained using the Roelands equation and assuming a compressible lubricant. Both figures show the well-known features for medium to highly loaded EHL contacts. The pressure profile approximates the Hertzian dry contact semiellipsoid, significantly deviating from it only in the inlet region of pressure build-up and in the region preceding the cavitated zone, where the pressure spike occurs.

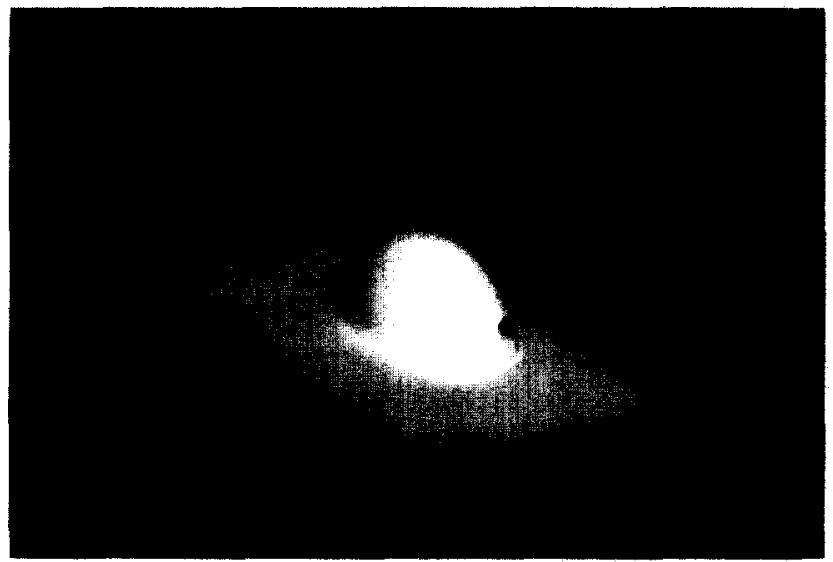

Fig. 1. Dimensionless pressure $P$ as a function of $X$ and $Y$ for $M=1000, L=10$ and $D=0.4$

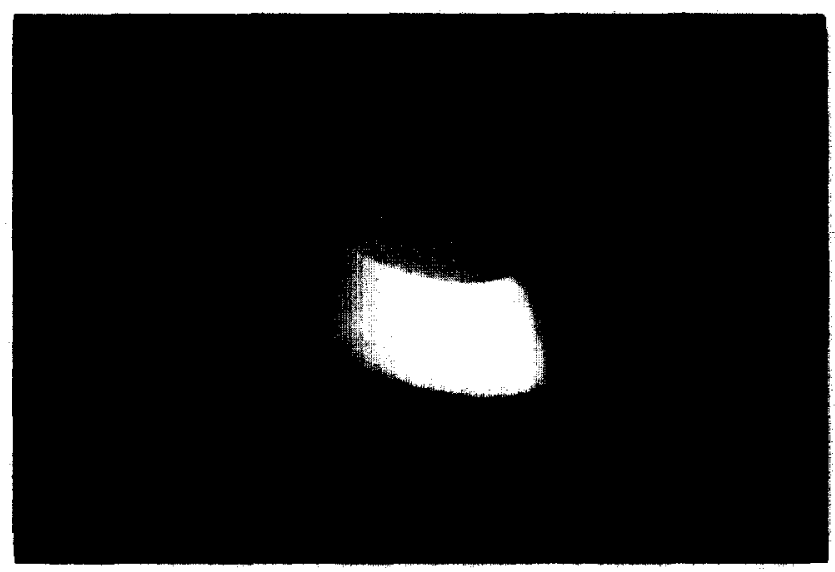

Fig. 2. Dimensionless film thickness $H$ as a function of $X$ and $Y$ for $M=1000, L=10$ and $D=0.4$.

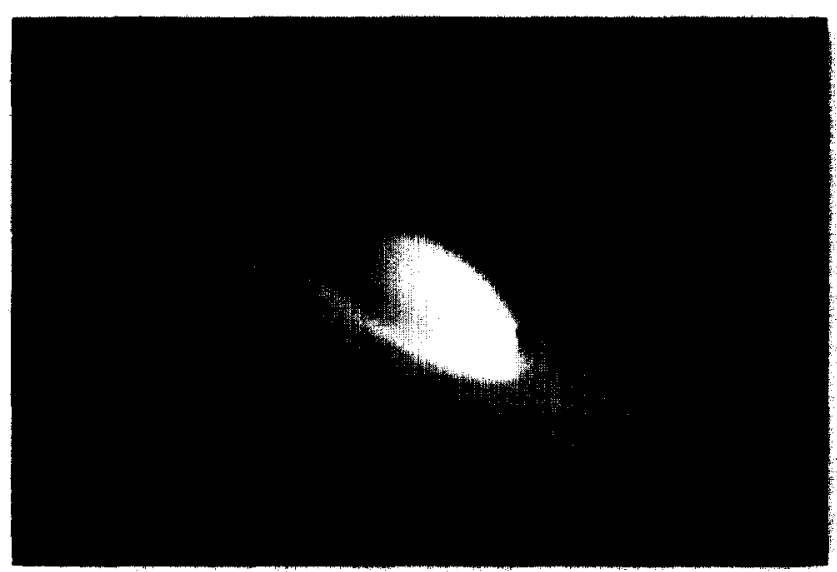

Fig. 3. Dimensionless pressure $P$ as a function of $X$ and $Y$ for $M=1000, L=10$ and $D=0.2$.

The film thickness is nearly uniform in the centre, shows a horseshoe-shaped restriction on the downstream side of the contact, and reaches its minimum value in the so-called side-lobes near $Y= \pm 1 / \kappa$. Note that the size of these side-lobes, relative to the size of the contact, is smaller for $D=0.2$ than for $D=0.4$. 


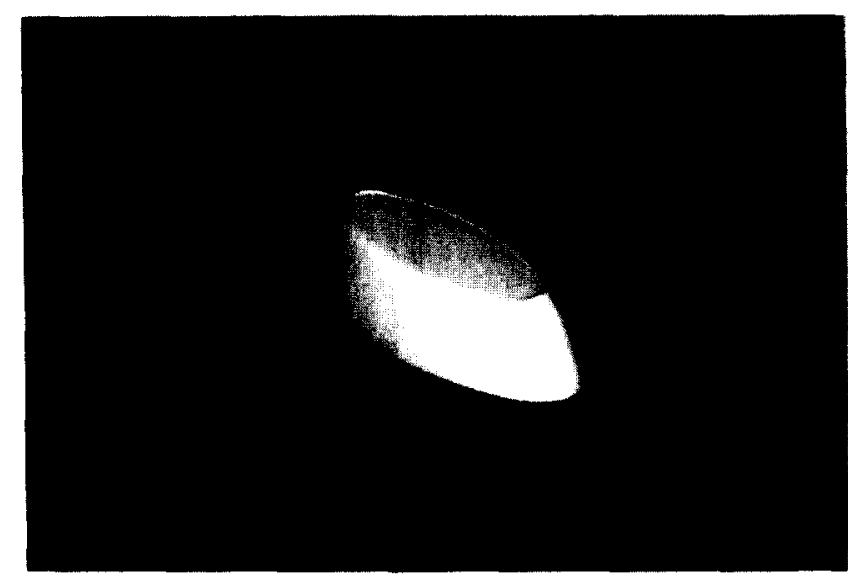

Fig. 4. Dimensionless film thickness $H$ as a function of $X$ and $Y$ for $M=1000, L=10$ and $D=0.2$.

\subsection{Varying load and lubricant parameter}

Pressure profiles and associated film thickness maps were computed for a wide range of operating conditions and for two curvature ratios, i.e. $D=0.4$ and $D=0.2$. Figs. 5 and 6 show the contour plots of the film thickness for a selection of solutions of varying $M$ and $L$ (obtained using Roelands' equation and a compressible lubricant). These contour plots are a convenient way to show the changes in the film thickness with changing $M$ and $L$. Both Fig. 5 and Fig. 6 show the same tendencies as observed for the circular contact (see Ref. [11], Fig.
9.13, and Ref. [24], Fig. 7). With increasing $M$ and $L$ the film profile in the centre of the contact flattens and side-lobes are formed. For low $M$ and $L$ the overall minimum film thickness occurs near the exit at the centre-line of the contact. However, once the side-lobes have formed, they become the location where the minimum film thickness occurs. As a result the ratio between the central and minimum film thicknesses is not a constant but depends on the load, as will be discussed in more detail in section 6 .

Note that, compared with the solutions with $D=0.4$, larger $M$ and $L$ are needed for the side-lobes to appear when $D=0.2$. Similarly, in contacts with $D=0.4$, larger $M$ and $L$ are required for the side-lobes to appear than for $D=1.0$ (circular contact). A likely explanation for this behaviour is that the formation of the sidelobes is related to the elastic deformation of the surfaces and thereby to the maximum Hertzian pressure $p_{\mathrm{h}}$. The side-lobes will just show up once the deformation is sufficiently large, i.e. once $p_{\mathrm{h}}$ exceeds a certain level. From Appendix $\mathrm{C}$ it can be inferred that $p_{\mathrm{h}}$ increases with increasing $M$ and $L$ and decreases with decreasing $D$. Therefore, to reach the same level of (maximum Hertzian) pressure and deformation, and thus for the side-lobes to appear, larger $M$ and $L$ are required for smaller $D$.

Focusing on the central region of the contact, Figs. 5 and 6 show that a larger ellipticity of the contact causes an increased region of nearly uniform film thick-
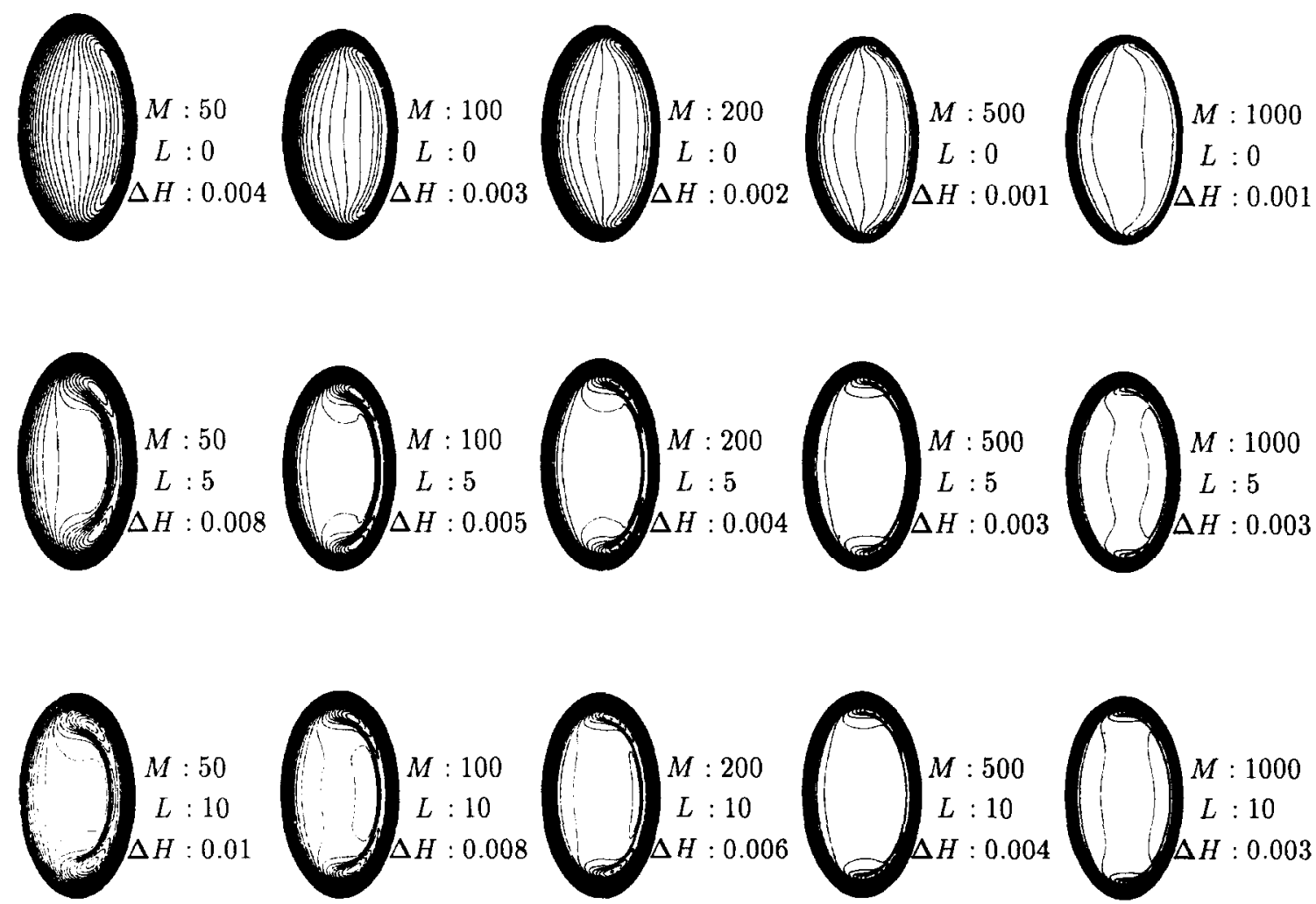

Fig. 5. Contour plots of the dimensionless film thickness $H$ for $D=0.4$. 

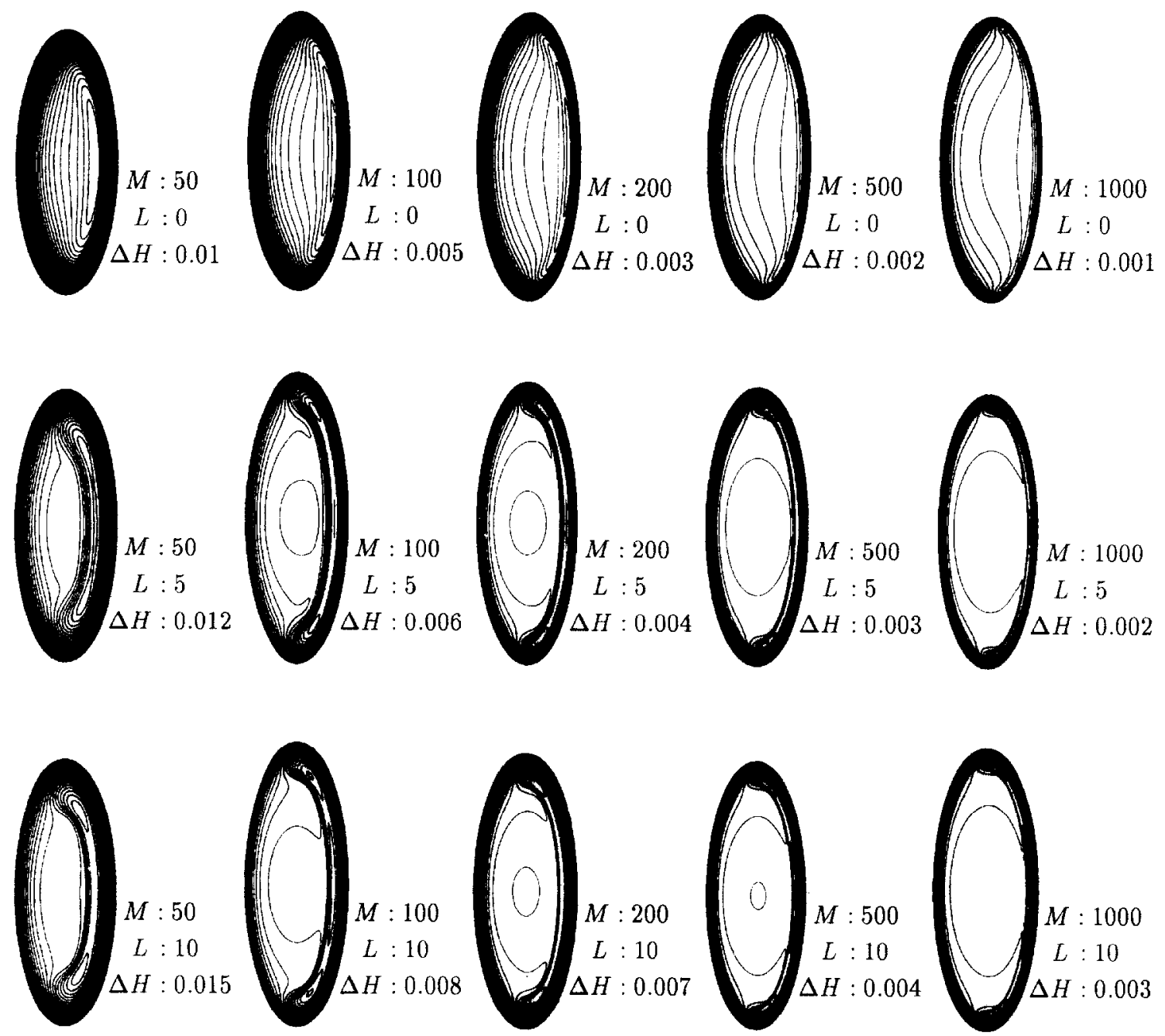

Fig. 6. Contour plots of the dimensionless film thickness $H$ for $D=0.2$.

ness. This is indicated by the low density of contour lines in this region. Furthermore, by taking a closer look an additional subtle change can be observed. When $D=0.4$, as for the circular contacts $(D=1)[11,24]$, the contour lines near the centre are concave with respect to the line $Y=0$. This implies that the film thickness decreases with increasing $Y$. However, for the same $M$ and $L$ but $D=0.2$, the contour lines in the centre of the contact may become elliptical. This suggests the occurrence of a local minimum in the film thickness at $X=0$ and $Y=0$. This phenomenon is investigated in more detail in the next section.

\subsection{Influence of $D$}

For one set of parameters, i.e. $M=500$ and $L=5$, the curvature ratio $D$ is varied. Fig. 7 shows the film thickness as a function of $Y$ on the line $X=0$ for $D=1$, $0.4,0.2$ and 0.1 . These graphs confirm what is suggested by the contour plots, i.e. below a certain value of $D$ the film thickness shows a local minimum in the centre of contact.

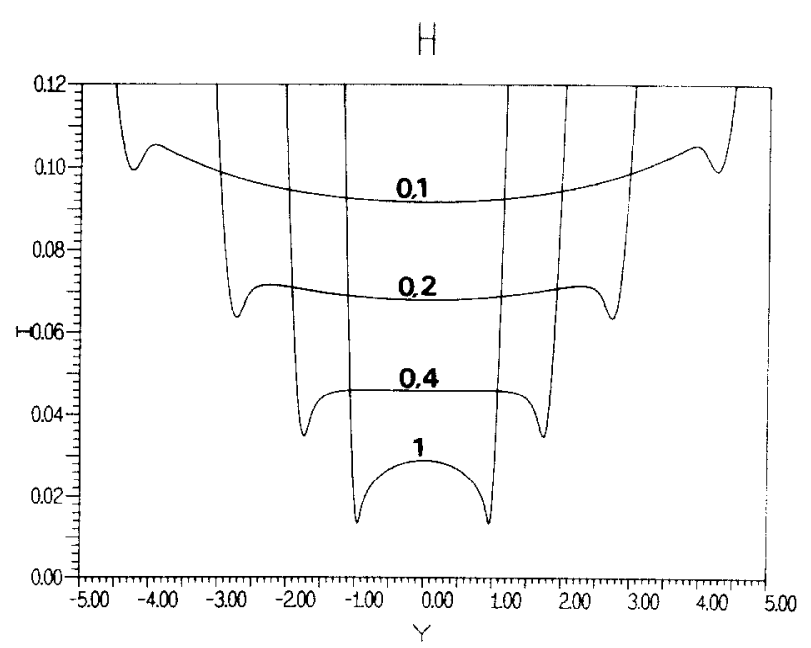

Fig. 7. Dimensionless film thickness $H$ as a function of $Y(X=0)$ for $M=500, L=5$ and $D=1,0.4,0.2$ and 0.1 .

The occurrence of this local minimum can be explained by the influence of the compressibility. As explained above, by fixing $M$ and $L$ the contact load 
is fixed. Consequently, decreasing $D$ implies a decreasing maximum (Hertzian) pressure. For example the maximum Hertzian contact pressure for the cases shown in Fig. 7 decreases from $0.66 \mathrm{GPa}(D=1)$ to about $0.35 \mathrm{GPa}(D=0.1)$. Now, as long as the pressure in the major part of the central region exceeds the level where Dowson and Higginson's equation allows significant density changes, the contact form will remain the same as for $D=1$. However, once the pressure drops into the range where pressure variations cause significant density variations, this local minimum will show up. This phenomenon is rooted in the fact that, owing to a small (and for sufficiently large loads even negligible) pressure flow, the Reynolds equation in this region enforces $\bar{\rho} H \approx c(Y)$, as a result of which density changes tend to be fully compensated by film changes. For a detailed explanation the reader is referred to Venner and Bos [25].

\subsection{Equivalent line contact}

For the class of contacts considered here, i.e. $D \leqslant 1$, with increasing ellipticity, i.e. decreasing $D$, the lubricant flow perpendicular to the entraining velocity (sideleakage) will decrease. Consequently for sufficiently small values of $D$, at least at the centre-line $(Y=0)$, one may intuitively expect the solution to approximate the solution as predicted by a line contact type of analysis. This expectation is investigated below. First for a given elliptical contact an equivalent line contact is defined by taking the same maximum Hertzian pressure and half-width of the contact. In that way, from the dimensionless parameters $M, L$, and $D$ characterising the elliptical contact, dimensionless line contact parameters $M_{1}$ and $L$ characterising the equivalent line contact are obtained, see Appendix D. For the same set of solutions as presented in section 5.2, Fig. 8 displays the dimensionless minimum and central film thickness together with the values as obtained from solving the equivalent line contact problem using the algorithm presented in Ref. [26].

Starting out from a circular contact $(D=1)$, both the minimum and the central film thickness increase with decreasing $D$. As could be anticipated, because sideleakage is neglected, the equivalent line contact prediction overestimates the film thickness, particularly its minimum value (which for these point contact cases occurs in the side-lobes). However, with decreasing $D$ the difference between the elliptical and the line contact values decreases and from $D \approx 0.1$ onwards, the prediction from the equivalent line contact becomes very accurate. This is, for example, illustrated in Fig. 9, which shows the pressure profile and film thickness on the centre-line of the contact for $D=0.1$, and also the pressure profile and film thickness as obtained from numerically solving the equivalent line problem for $M_{1}$ and $L_{1}$. Obviously both results closely agree.

At this point it is noted that the onset of this close agreement between the equivalent line contact predictions and the actual point contact results coincides with the disappearance of the side-lobes. As a result, the minimum film thickness in the point contact occurs, as in a line contact, at the centre-line near the exit. Consequently, below this onset value of $D$, the ratio between central and minimum film thickness assumes values obtained for the line contact problem. (1.2 for the present loading case using Dowson and Higginson's

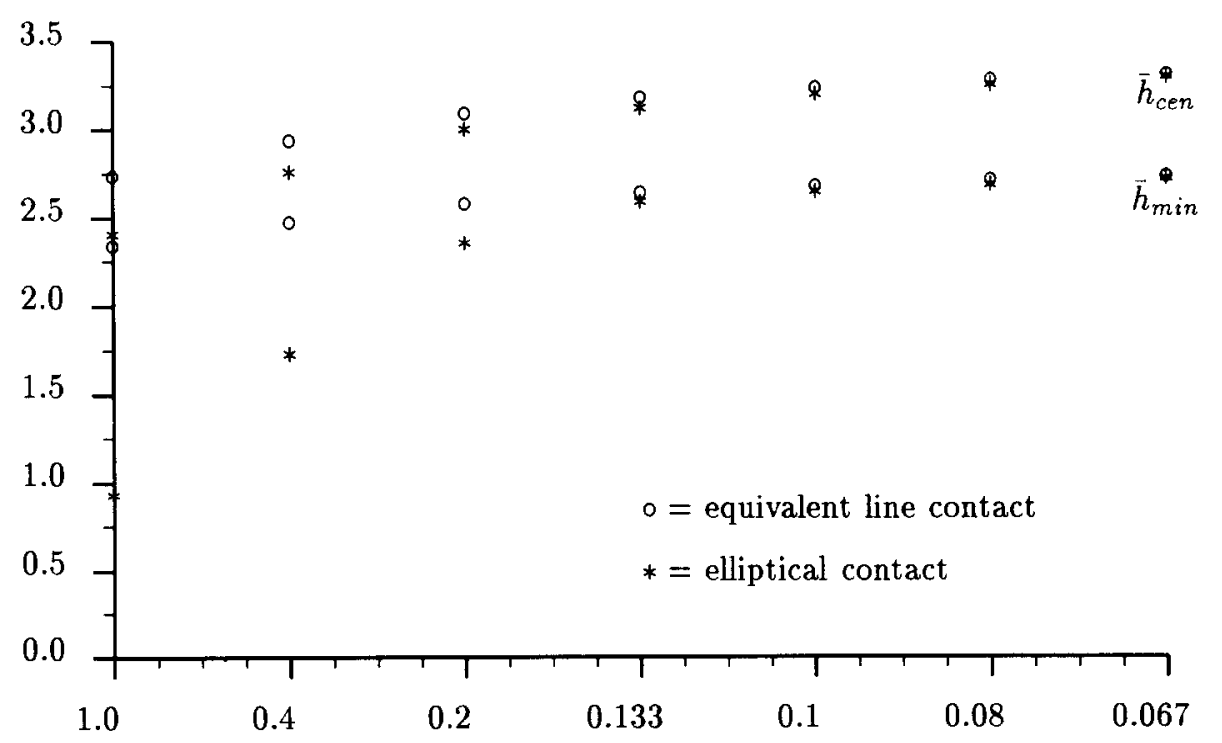

Fig. 8. Dimensionless minimum $\left(h_{\min }\right)$ and central film thickness $\left(h_{\mathrm{cen}}\right)$ as functions of $D$ obtained for the full elliptic contact solution, and the values obtained from solving the equivalent line contact problem $(M=500, L=5)$. 


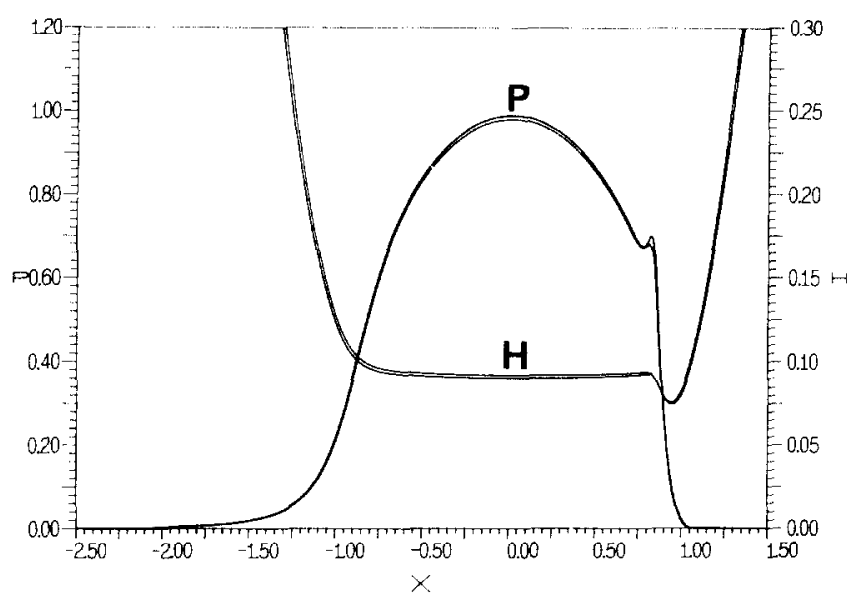

Fig. 9. Dimensionless film thickness $H$ and dimensionless pressure profile $P$ as functions of $X(Y=0)$ for the full elliptic contact solution, and the result obtained by solving the equivalent line contact problem $(M=500, L=5, D=0.067)$.

equation and $4 / 3$ for an incompressible lubricant, see also Ref. [26]).

Finally, the derivation of the equivalent line contact parameters uses results from the Hertzian contact theory. Consequently predictions based on this equivalent line contact will be poor in regions of small deformation, i.e. near the rigid isoviscous regime (asymptote). As a rough estimate, it can be said that the concept of an equivalent line contact with $M$ as given here should only be used for $M \geqslant 20$.

\section{Film thickness survey diagrams}

In the previous section the changes of the film thickness map with varying load, lubricant and ellipticity parameter were investigated. Next, as a first step towards a film thickness formula, calculated values of dimensionless minimum and central film thickness are presented in survey diagrams. To facilitate comparison with existing film thickness formulae and asymptotic solutions these results were computed using Barus' equation and assuming an incompressible lubricant.

First Figs. 10 and 11 display the dimensionless minimum film thickness as function of $M$ and $L$ for $D=0.4$ and $D=0.2$. The most extreme load situation represented in the diagrams is $M=1000, L=25$. This results in a maximum Hertzian contact pressure of $p_{\mathrm{h}}=3.1$ $\mathrm{GPa}$ for $D=0.4$, and $p_{\mathrm{h}}=2.6 \mathrm{GPa}$ for $D=0.2$. The lines drawn in these figures indicate the values that can be obtained from an analysis assuming rigid surfaces and an isoviscous lubricant (see section 7, Eq. (4)).

Figs. 12 and 13 present the central film thickness as function of $M$ and $L$ for $D=0.4$ and $D=0.2$. The broken lines in the figures represent the central film thickness as predicted by the formula of Chittenden et al. [2]. Notice that the predictions agree quite well with the numerical results in the range away from the asymptotic regions, i.e. in the range $M \geqslant 20,2.5 \leqslant L \leqslant 10$, although the slope with increasing load $M$ is too steep. The full curves in the Figs. 12 and 13 are the predictions of the central film thickness formula as described below, see section 7 .

A detailed comparison of Fig. 10 with Fig. 12 and of Fig. 11 with Fig. 13 shows that, as mentioned in section 5.1 , the ratio between the central and minimum film thicknesses in a point contact is not a constant but depends on the load. For the computational results presented in Figs. 10-13 this is shown in more detail in Table 3. For relatively low $M$ and $L$ the ratio is about $4 / 3$, which indicates that the minimum film thickness appears on the centre-line near the exit, see section 5.3. For larger values of $M$ and $L$ the ratio between the central and the minimum film thickness increases with increasing load, the onset of this behaviour being the appearance of the side-lobes. Consequently for deviations from this value to occur for the smaller value of $D$ requires larger $M$ and $L$, see also section 5.1.

One of the objectives of the present study is to obtain a film thickness formula for practical use. The question then arises what film thickness value should be used to characterise the contact, the minimum or the central film thickness. In many cases the aim in design is to achieve full separation of the surfaces. Generally this is assumed to be ensured if the minimum film thickness predicted by the smooth surface analysis is sufficiently large compared with the combined roughness of the surfaces. For the line contact problem this choice of the minimum film thickness as a selection criterion is quite obvious, i.e. because the problem is one dimensional, any feature present on one of the surfaces will have to pass the location of the minimum film thickness. Besides, also because the problem is one dimensional, once the minimum film thickness is known, the central film thickness can be computed and vice versa as the mass flow through the contact roughly fixes the ratio between these two film thicknesses at $4 / 3$ for the incompressible case and at this value divided by $\bar{\rho}\left(p_{h}\right)$ if a compressible lubricant is assumed.

However, in the case of a point contact, using the minimum film thickness is no longer obvious. In that case the film thickness is roughly uniform at the value of the central film thickness throughout the major part of the Hertzian contact region. The film thickness drops below this value only in the side-lobes (highly loaded contacts) or near the exit (lowly loaded contacts or wide contact ellipses). In the first case obviously the side-lobes cover only a small part of the total contact region and because of their location the chances that a particular surface feature will have to pass the minimum film thickness are relatively small. Hence in these cases the central film thickness may be a more realistic parameter to characterise the contact. 


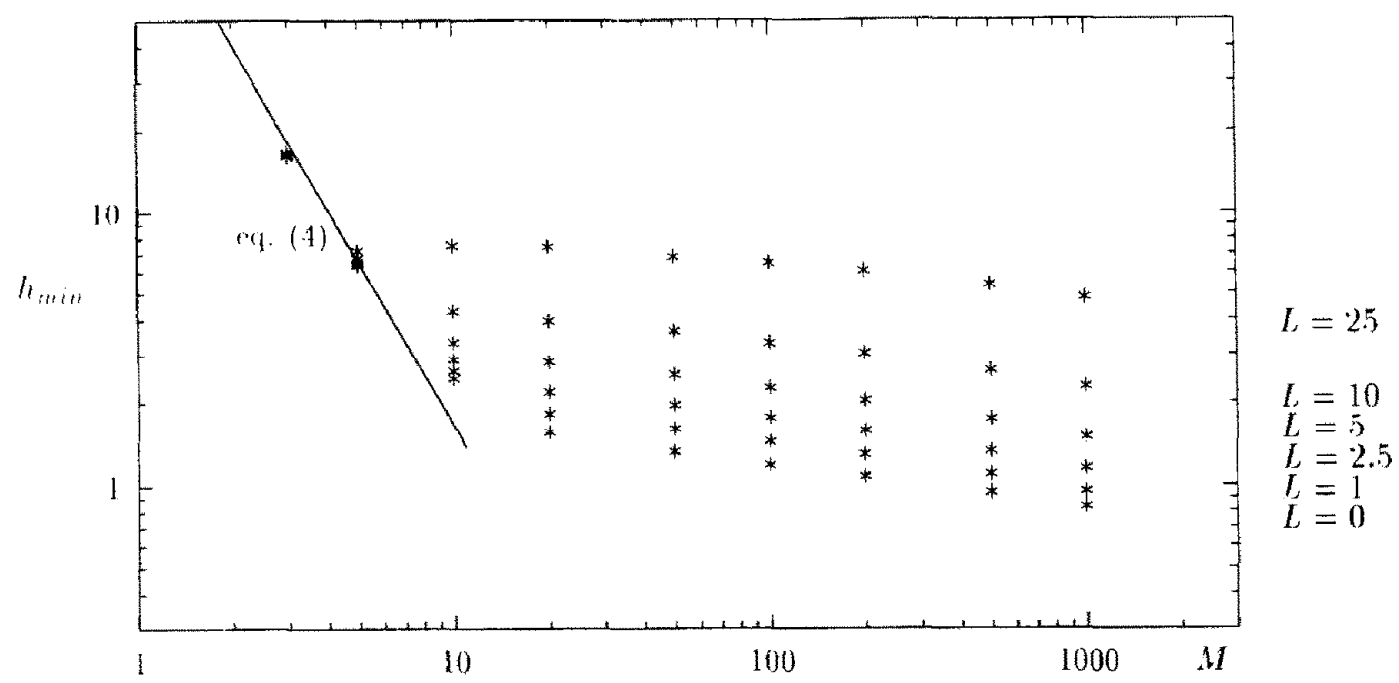

Fig. 10. Survey diagram of the dimensionless minimum film thickness $h_{\min }$ as a function of $M$ and $L$ for $D=0.4$.

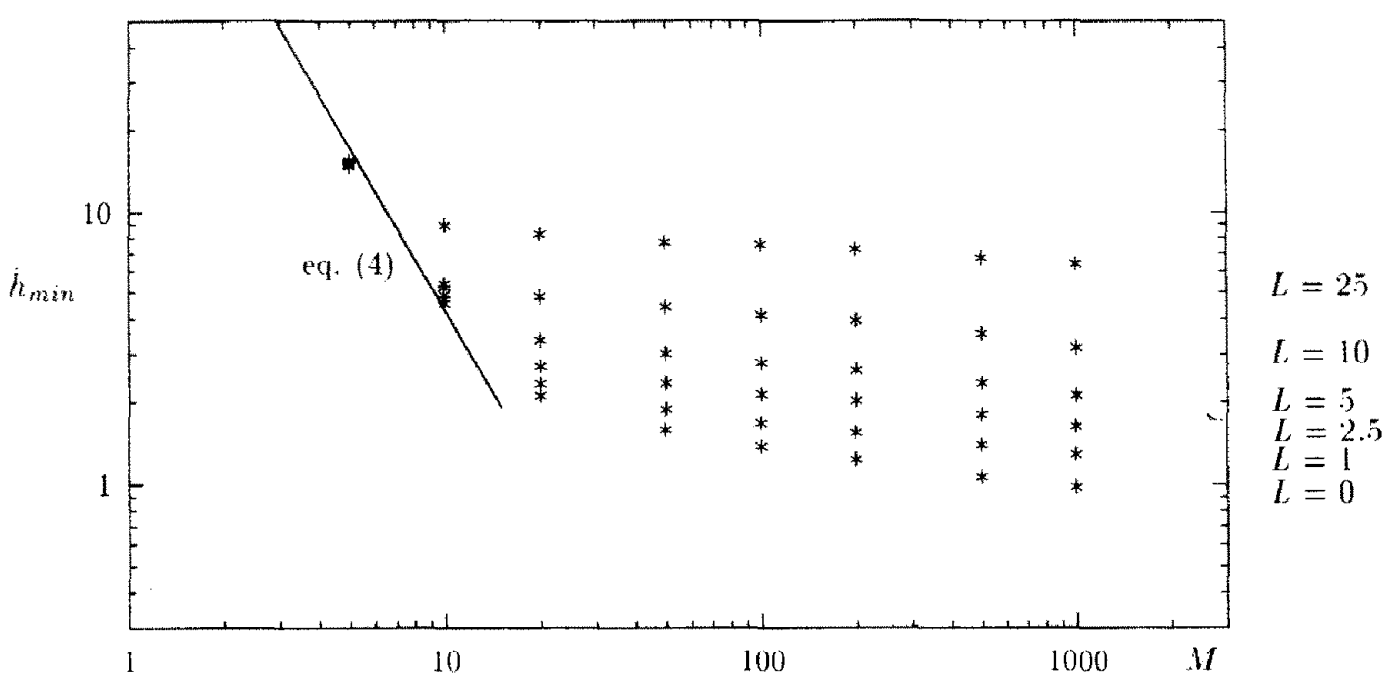

Fig. 11. Survey diagram of the dimensionless minimum film thickness $h_{\min }$ as a function of $M$ and $L$ for $D=0.2$.

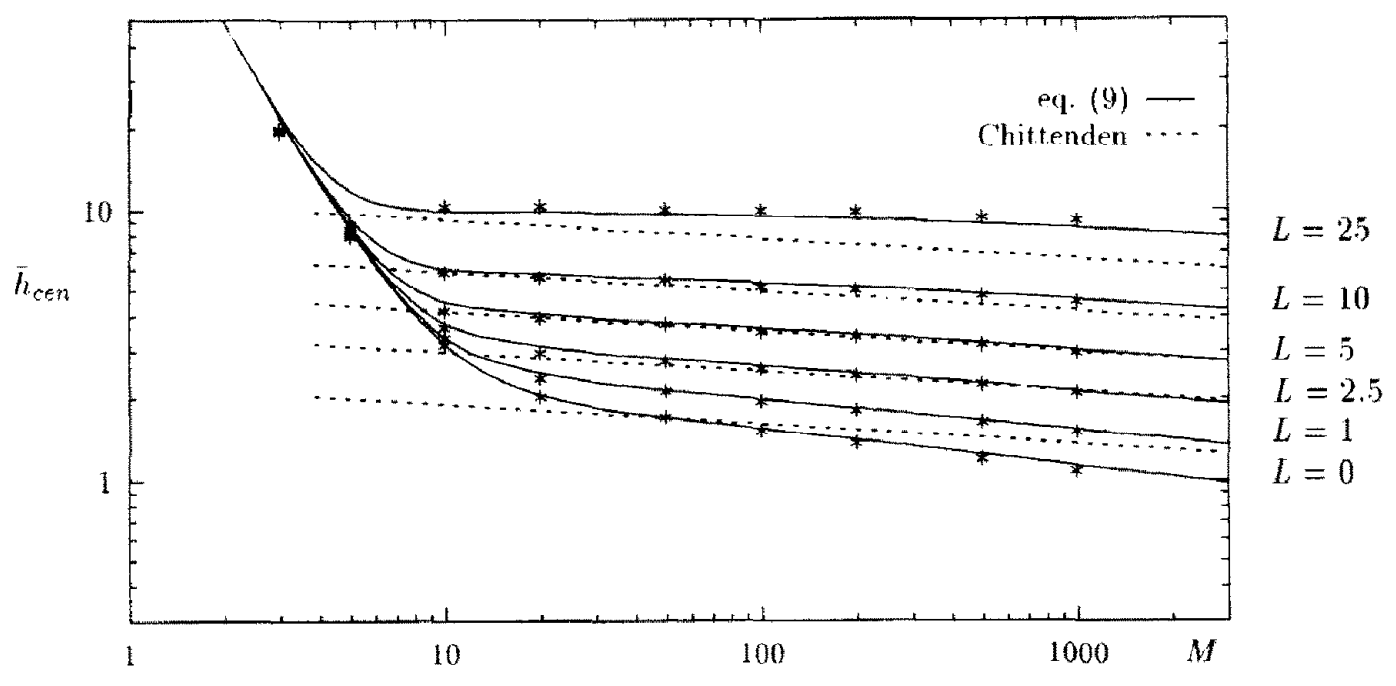

Fig. 12. Survey diagram of the dimensionless central film thickness $h_{c e n}$ as a function of $M$ and $L$ for $D=0.4$. The broken curves represent the predictions of Chittenden et al. [2]. The full curves represent the predictions of Eq. (9). 


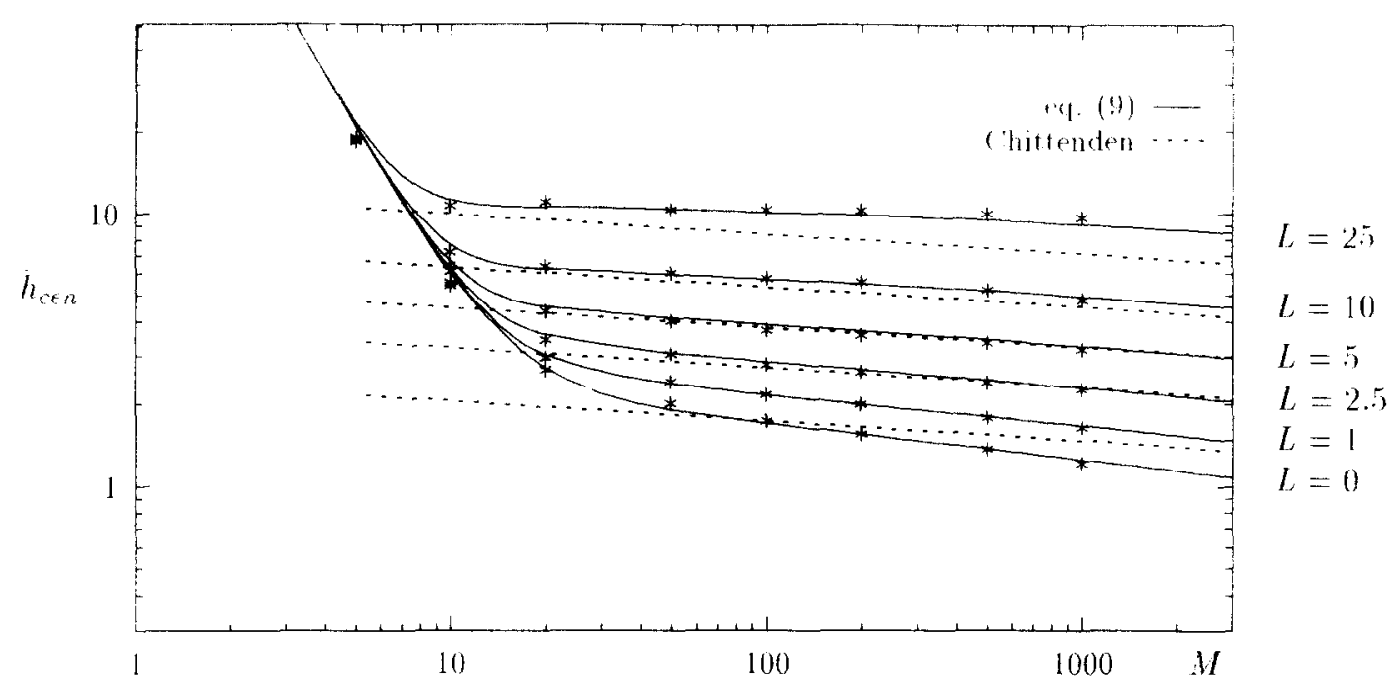

Fig. 13. Survey diagram of the dimensionless central film thickness $h_{\text {cen }}$ as a function of $M$ and $I$, for $D=0.2$. The broken curves represent the predictions of Chittenden et al. [2]. The full curves represent the predictions of Eq. (9).

Table 3

Ratio $\bar{h}_{\text {cen }} / \bar{h}_{\min }$ as a function of $M$ and $L$ for $D=0.4$ and $D=0.2$

\begin{tabular}{|c|c|c|c|c|c|c|c|c|}
\hline \multicolumn{9}{|l|}{$M$} \\
\hline L & 5 & 10 & 20 & 50 & 100 & 200 & 500 & 1000 \\
\hline \multicolumn{9}{|c|}{$D=0.4$} \\
\hline 0 & 1.3 & 1.3 & 1.3 & 1.3 & 1.3 & 1.3 & 1.3 & 1.3 \\
\hline 1 & 1.3 & 1.3 & 1.3 & 1.3 & 1.4 & 1.4 & 1.5 & 1.6 \\
\hline 2.5 & 1.3 & 1.3 & 1.3 & 1.4 & 1.5 & 1.6 & 1.7 & 1.8 \\
\hline 5 & 1.3 & 1.3 & 1.4 & 1.5 & 1.5 & 1.6 & 1.8 & 2.0 \\
\hline 10 & 1.3 & 1.4 & 1.4 & 1.5 & 1.5 & 1.6 & 1.8 & 2.0 \\
\hline 25 & - & 1.4 & 1.3 & 1.5 & 1.6 & 1.6 & 1.7 & 1.9 \\
\hline \multicolumn{9}{|c|}{$D=0.2$} \\
\hline 0 & 1.2 & 1.3 & 1.3 & 1.3 & 1.3 & 1.3 & 1.3 & 1.3 \\
\hline 1 & 1.2 & 1.3 & 1.3 & 1.3 & 1.3 & 1.3 & 1.3 & 1.4 \\
\hline 2.5 & 1.2 & 1.3 & 1.3 & 1.3 & 1.3 & 1.3 & 1.3 & 1.4 \\
\hline 5 & 1.2 & 1.3 & 1.3 & 1.3 & 1.3 & 1.4 & 1.4 & 1.5 \\
\hline 10 & 1.2 & 1.3 & 1.3 & 1.4 & 1.4 & 1.4 & 1.5 & 1.5 \\
\hline 25 & - & 1.3 & 1.3 & 1.4 & 1.4 & 1.4 & 1.5 & 1.5 \\
\hline
\end{tabular}

In the second case, i.e. low load or wide contact ellipse, as for a line contact, almost any feature will have to pass a region with a film thickness close to the minimum film thickness. However, for these wide contact ellipses, owing to the small side-leakage, the central and minimum film thickness are coupled again by the mass flow as described above for the line contact problem. Hence, if in such cases the central film thickness is given, the minimum value can be obtained easily.

Based on these arguments the authors have chosen to derive a formula giving the central film thickness as a function of load, lubricant parameters and the curvature ratio.

\section{Film thickness formula}

For the film thickness in a point contact the following asymptotic regimes can be distinguished.

\subsection{Rigid isoviscous (small $M$, and $L=0$ )}

For this regime a straightforward similarity analysis yields

$\bar{h}_{\min }=C_{\mathrm{m}}^{\mathrm{RI}}(D) M^{-2}$

and

$\bar{h}_{\mathrm{cen}}=C_{\mathrm{c}}^{\mathrm{RI}}(D) M^{-2}$

The values of $C_{\mathrm{m}}^{\mathrm{RI}}$ and $C_{\mathrm{c}}^{\mathrm{RI}}$ as functions of $D$ remain to be determined. Based on the work of Kapitza [27] Brewe et al. [28], and numerical solutions of the rigid isoviscous point contact equations for various values of $D$ performed in the course of the present research, it was found that the following function fit solution applies:

$C_{\mathrm{c}}^{\mathrm{RI}} \approx 145\left(1+0.796 D^{14 / 15}\right)^{-15 / 7} D^{-1}$

and $C_{\min }^{\mathrm{RI}}=(3 / 4)_{\mathrm{c}}^{\mathrm{RI}}$.

\subsection{Elastic isoviscous (large $M$, and $L=0$ )}

This solution is characteristic of elastomeric seals. Based on the work of Hamrock and Dowson [29], Crook [30], and Kanters [31], the following formal expression may be derived for the central film thickness in this regime:

$\bar{h}_{\mathrm{cen}}=C_{\mathrm{cen}}^{\mathrm{EI}}(D) M^{-2 / 15}$

with the function fit solution

$C_{\mathrm{cen}}^{\mathrm{EI}}=3.18\left(1+0.006 \ln D+0.63 D^{4 / 7}\right)^{-14 / 25} D^{-1 / 15}$

\subsection{Rigid piezoviscous (small M, large L)}

For this regime the following formal equation was derived by Grubin [32]: 
$\bar{h}_{\text {cen }}=C_{\text {cen }}^{\mathrm{RP}}(D) L^{2 / 3}$

with the function fit solution

$C_{\text {cen }}^{\mathrm{RP}}=1.29(1+0.691 D)^{-2 / 3}$

This solution was based on the asymptotic solutions for $D \rightarrow 0$ and $D \rightarrow \infty$ as well as on numerical calculations for $D \approx 1$.

\subsection{Elastic piezoviscous (large $M$, large $L$ )}

The following formal equivalent of the Grubin solution [32] may be derived for elliptic contacts:

$\bar{h}_{\text {cen }}=C_{\text {cen }}^{\mathrm{EP}}(D) M^{-1 / 12} L^{3 / 4}$

with the function fit solution

$C_{\mathrm{cen}}^{\mathrm{EP}}=1.48\left(1+0.006 \ln D+0.63 D^{4 / 7}\right)^{-7 / 20} D^{-1 / 24}$

\subsection{Formula}

Introducing $\bar{h}_{\mathrm{RI}}, \bar{h}_{\mathrm{EI}}, \bar{h}_{\mathrm{RP}}$ and $\bar{h}_{\mathrm{EP}}$ for the film thickness values predicted by Eqs. (5), (6), (7) and (8), respectively, the following function fit formula for the dimensionless central film thickness is proposed:

$$
\begin{aligned}
\bar{h}_{\mathrm{cen}}= & \left\{\left[\bar{h}_{\mathrm{RIc}}^{3 / 2}+\left(\bar{h}_{\mathrm{EIc}}^{-4}+\bar{h}_{00}^{-4}\right)^{-3 / 8}\right]^{2 s / 3}\right. \\
& \left.+\left(\bar{h}_{\mathrm{RPc}}^{-8}+\bar{h}_{\mathrm{EPc}}^{-8}\right)^{-\mathrm{s} / 8}\right\}^{1 / s}
\end{aligned}
$$

with

$$
\begin{aligned}
& s=1.5\left[1+\exp \left(-1.2 \frac{\bar{h}_{\text {EIc }}}{\bar{h}_{\text {RIc }}}\right)\right] \\
& \bar{h}_{00}=1.8 D^{-1}
\end{aligned}
$$

The last two parameters serve to ensure smooth transitions according to the numerical calculations between the four asymptotic solutions. The values predicted by Eq. (9) for $D=0.4$ and $D=0.2$ are shown in the Figs. 12 and 13 by the full curves. From these figures it can be seen that $\mathrm{Eq}$. (9) accurately predicts the dimensionless central film thickness also in the asymptotic regimes. Notice that Eq. (9) is in fact a generalisation of the film thickness formula presented by Venner and ten Napel [24] for $D=1$, i.e. for circular contacts.

\section{Conclusions}

A multilevel algorithm for the smooth EHL circular contact problem has been extended to elliptical contact problems and applied in a parametric study of contacts with $D=R_{x} / R_{y} \leqslant 1$, i.e. to contacts with the entrainment direction perpendicular to the major principal axis of the contact ellipse. The variations of pressure profile and particularly of the film thickness with the load parameters and ellipticity of the contact were studied. Also it was investigated when the film thickness on the centre-line of elliptical contact can be approximated with an equivalent line contact. For two curvature ratio solutions of the minimum and central film thicknesses are displayed in survey diagrams. With the aid of these results, a film thickness equation was derived for elliptical contacts. Because this formula incorporates asymptotic behaviour it is valid for all load conditions. In this paper it is shown to be very accurate for elliptical contacts with $D \leqslant 1$, leaving its accuracy for contacts with $D>1$ to be investigated in future research.

\section{References}

[1] B.J. Hamrock and D. Dowson, Isothermal elastohydrodynamic lubrication of point contacts. Part 2 - ellipticity parameter results, ASME JOT, 98 (1976) 375-383.

[2] R.J. Chittenden, D. Dowson, J.F. Dunn and C.M. Taylor, A theoretical analysis of the isothermal elastohydrodynamic lubrication of concentrated contacts 2 . General case, with lubricant entrainment along either principal axis of the Hertzian contact ellipse or at some intermediate angle, Proc. R. Soc. London, Ser. A, 397 (1985) 271-294.

[3] J. Seabra and D. Berthe, Elastohydrodynamic point contacts. Part I: Formulation and numerical solution, Wear, 130 (1989) 301-318.

[4] J. Seabra and D. Berthe, Elastohydrodynamic point contacts. Part I: Influence of surface speeds, surface waviness, and load on the contact behaviour, Wear, 130 (1989) 319-335.

[5] C.C. Kweh, H.P. Evans and R.W. Snidle, Micro-elastohydrodynamic lubrication of an elliptical contact with transverse and three-dimensional sinusoidal roughness, $A S M E$ JOT, 111 (1989) 577-584.

[6] Fd.M. Barragan de Ling, H.P. Evans and R.W. Snidle, Micro-elastohydrodynamic lubrication of circumferentially finished rollers: the influence of temperature and roughness, ASME JOT, 111 (1989) 730-736.

[7] H.P. Evans and R.W. Snidle, Wildhaber-Novikov circular arc gears: Elastohydrodynamics, ASME JOT, 115 (1993) $487-492$.

[8] R.J. Chittenden, D. Dowson and C.M. Taylor, The lubrication of elliptical conjunctions in the isoviscous-elastic regime with entrainment directed along either principal axis, Proc. 1986 Leeds-Lyon Symp. on Tribology, 1986, pp. 247-260.

[9] C.I. Hooke, Calculation of clearances in soft point contacts, ASME JOT, 110 (1987) 167-173.

[10] C.J. Hooke, An interpolation procedure for the minimum film thickness in point contacts, Proc. Inst. Mech. Eng., 204 (1990) 199-206.

[11] C.H. Venner, Multilevel solution of the EHL line and point contact problems, Ph.D. Thesis, University of Twente, Enschede, The Netherlands (ISBN 90-9003974-0).

[12] O. Reynolds, On the theory of lubrication and its application to Mr Beauchamp Tower's experiments, including an experimental determination of the viscosity of olive oil, Philos. Trans. R. Soc., London, 177 (1886) 157-234.

[13] A.E.H. Love, A treatise on the mathematical theory of elasticity, Dover Publications, New York, 4th edn., 1944.

[14] C. Barus, Isothermals, isopiestics and isometrics relative to viscosity, Am. J. Sci. 45 (1893) 87-96. 
[15] C.J.A. Roelands, Correlational aspects of the viscositytemperature-pressure relationship of lubricating oils, Ph.D. Thesis, Technische Hogeschool Delft, Netherlands.

[16] D. Dowson and G.R. Higginson, Elasto-hydrodynamic lubrication. The fundamentals of roller and gear lubrication, Pergamon, Oxford, 1966.

[17] H. Hertz, Über die Berührung fester elastischer Körper, Z. reine angew. Math., 92 (1881) 156-171 (in German).

[18] H. Moes, Optimum similarity analysis with applications to elastohydrodynamic lubrication, Wear, 159 (1992) 56-66.

[19] C.H. Venner and W.E. ten Napel, Multilevel solution of the elastohydrodynamically lubricated circular contact problem. Part I: theory and numerical algorithm, Wear, 152 (1992) 351-367.

[20] W.L. Briggs, A Multigrid Tutorial, SIAM, Philadelphia, PA, 1987.

[21] A. Brandt, Multigrid Techniques: 1984 Guide with Applications to Fluid Dynamics, 1984, available as G.M.D.-Studien No. 85, from G.M.D.-FIT, Postfach 1240, D5205, St. Augustin 1 , Germany.

[22] A.A. Lubrecht, The numerical solution of the elastohydrodynamically lubricated line- and point contact problem using multigrid techniques, Ph.D. Thesis, Universiteit Trwente, Enschede, The Netherlands (ISBN 90-9001583-3).

[23] A. Brandt and A.A. Lubrecht, Multilevel matrix multiplication and fast solution of integral equations, J. Comput. Phys., 90 (1990) (2) 348-370.

[24] C.H. Venner and W.E. ten Napel, Multilevel solution of the elastohydrodynamically lubricated circular contact problem. Part II: smooth surface results, Wear, 152 (1992) 369-381.

[25] C.H. Venner and J. Bos, Effects of lubricant compressibility on the film thickness in EHL linc and circular contacts, Internal Rep. WB.93/TR-287 (University of Twente), to appear in Wear.

[26] C.H. Venner, W.E. ten Napel and R. Bosma, Advanced multilevel solution of the EHL line contact problem, $A S M E$ JOT, 112 (1990) 426-432.

[27] P.L. Kapitza, Hydrodynamic theory of lubrication during rolling. Zh. Tekh. Fiz., 25 (1955) 747-162 (in Russian).

[28] D.E. Brewe, B.J. Hamrock and C.M. Taylor, Effect of geometry on hydrodynamic film thickness, ASME JOT, 101 (1979) 231-239.

[29] B.J. Hamrock and D. Dowson, Elastohydrodynamic lubrication of elliptical contacts for material of low elastic modulus 1 - Fully flooded conjunction, ASME JOT, 100 (1978) 236-245.

[30] A.W. Crook, The lubrication of rollers - II. Film thickness with relation to viscosity and speed, Philos. Trans. R. Soc., London, Ser. A, 254 (1961) 223-258.

[31] A.F.C. Kanters, On the calculation of leakage and friction of reciprocating elastomeric seals, Ph.D. Thesis, Technical University Eindhoven, Eindhoven, The Netherlands.

[32] A.N. Grubin, Fundamentals of the hydrodynamic theory of lubrication of heavily loaded cylindrical surfaces, in Kh.F. Ketova (ed.), Investigations of the contact of machine components, Moscow: TsNIITMASh, (D.S.I.R., London, Translation 337), Book 30, pp. 115-166.

\section{Appendix A: Nomenclature}

half width Hertzian (line contact), $a=$ $a_{x}$

half-width Hertzian contact, $x$-direction, $a_{x}=\left(6 R_{x} F_{\kappa} E_{\mathrm{c}} / E^{\prime} \pi(1+D)\right)^{1 / 3}$

$a_{y} \quad$ half-width Hertzian contact, $y$-direction, $a_{y}=a_{x} /$ $\kappa$

C constant

$D$ ratio of reduced radii of curvature, $D=R_{x} / R_{y}$

$E^{\prime} \quad$ reduced modulus of elasticity, $2 / E^{\prime}=\left(1-\nu_{2}^{2}\right) /$ $E_{1}+\left(1-\nu_{2}^{2}\right) / E_{2}$

$E_{\mathrm{c}} \quad$ elliptic integral

$F \quad$ external load

$h \quad$ film thickness

$\bar{h}$ dimensionless film thickness (Moes), $\bar{h}=$ $h / R_{x}\left(E^{\prime} R_{x} / \eta_{0} u_{\mathrm{s}}\right)^{1 / 2}$

$\bar{h}_{00} \quad$ side-leakage factor

$H$ dimensionless film thickness, $H=h R_{x} / a_{x}{ }^{2}$

$H_{0} \quad$ integration constant

$\Delta H \quad H$ increment in contour plot

$L$ dimensionless lubricant parameter (Moes), $L=\alpha E^{\prime}\left(\eta_{0} u_{s} / E^{\prime} R_{x}\right)^{1 / 4}$

$M$ dimensionless load parameter (Moes), $M=$ $\left(F / E^{\prime} R_{x} 2\right)\left(\eta_{0} u_{\mathrm{s}} / E^{\prime} R_{x}\right)^{3 / 4}$

$M_{1}$ dimensionless load parameter (Moes), $M_{1}=\left(w / E^{\prime} R\right)\left(\eta_{\mathrm{o}} u_{\mathrm{s}} E^{\prime} R\right)^{-1 / 2}$

$n \quad$ number of grid points

$p \quad$ pressure

$p_{\mathrm{h}} \quad$ maximum Hertzian pressure

$P \quad$ dimensionless pressure

$R \quad$ reduced radius of curvature

$s \quad$ curve fit factor

$u_{\mathrm{s}} \quad$ sum velocity, $u_{\mathrm{s}}=u_{1}+u_{2}$

$w \quad$ external load per unit width

$y$ coordinate in rolling direction

$X, X^{\prime}$ dimensionless coordinate, $X=x / a_{x}, X^{\prime}=x^{\prime} / a_{x}$

$y \quad$ coordinate

$Y, Y^{\prime}$ dimensionless coordinate

$z \quad$ viscosity index (Roelands equation)

$\alpha \quad$ pressure-viscosity index

$\alpha \quad$ dimensionless parameter, $\bar{\alpha}=\alpha p_{\mathrm{h}}$

$\kappa \quad$ ellipticity ratio, $\kappa=a_{x} / a_{y}$

$\epsilon \quad$ coefficient in Reynolds equation, $\epsilon=\bar{\rho} H^{3} / \bar{\eta} \lambda$

$\lambda$ dimensionless speed parameter, $\lambda=6 \eta_{0} u_{s} R_{x} 2 /$ $a_{x} 3 p_{\mathrm{h}}$

$\eta \quad$ viscosity

$\eta_{0} \quad$ viscosity at ambient pressure

$\eta \quad$ dimensionless viscosity, $\bar{\eta}=\eta / \eta_{0}$

$\rho$ density

$\rho_{0} \quad$ density at ambient pressure

$\rho$ dimensionless density, $\bar{\rho}=\rho / \rho_{0}$

\section{Subscripts}

cen central (location $\partial P / \partial X=\partial P / \partial Y=0$ ) or related to a variable at this location

min minimum or related to a variable at this location $x \quad x$-direction

$y \quad y$-direction 


\section{Superscripts}

RI rigid isoviscous

EI elastic isoviscous

RP rigid piezoviscous

EP elastic piezoviscous

\section{Appendix B: Hertzian parameters}

The Legendre normal integral (complete elliptic integral) of the second kind, used in the Hertzian theory [17] is defined by

$E_{\mathrm{c}}=\int_{0}^{\pi / 2} \frac{\mathrm{d} \psi}{\sqrt{\cos ^{2} \psi+\kappa^{2} \sin ^{2} \psi}}$

In the present work the following approximation has been used:

$E_{\mathrm{c}} \approx 0.5 \pi \kappa^{2}\left(1+\frac{1-\kappa^{2}}{0.5 \pi \kappa^{2}}-0.25 \log \kappa\right)$

where $\kappa=a_{x} / a_{y}$ denotes the ellipticity of the Hertzian contact and can be obtained from the following approximation [17]:

$\kappa \approx\left(1+\sqrt{\frac{\log (16 / D)}{2 D}}-\sqrt{\log 4}+0.16 \log D\right)^{-1}$

For a given value of $D$, using $\kappa$ and $E_{\mathrm{c}}$ as given above, the half-width of the contact width in the $x$-direction $a_{x}$ is given by

$a_{x}=\left(\frac{6 R_{x} F \kappa E_{c}}{E^{\prime} \pi(1+D)}\right)^{1 / 3}$

\section{Appendix C: Parameters}

The dimensionless parameters $\alpha$ and $\lambda$ used in section 2.1 are related to the Moes [18] dimensionless parameters $M$ and $L$ in the following way:

$$
\begin{aligned}
& \bar{\alpha}=\frac{L}{\pi}\left(\frac{3 M \kappa}{2}\right)^{1 / 3}\left(\frac{(1+D) \pi}{4 E_{\mathrm{c}}}\right)^{2 / 3} \\
& \lambda=\left(\frac{128 \pi^{3}}{3 M^{4} \kappa^{4}}\right)^{1 / 3}\left(\frac{(1+D) \pi}{4 E_{\mathrm{c}}}\right)^{1 / 3}
\end{aligned}
$$

The relation between the dimensionless film thicknesses $h$ and $H$ is

$\bar{h}=(1.5 M \kappa)^{2 / 3}\left(\frac{(1+D) \pi}{4 E_{\mathrm{c}}}\right)^{-2 / 3} H$

\section{Appendix D: Definition of the equivalent line contact}

For a given elliptic contact the equivalent line contact is defined as the contact having a half-width $a=a_{x}$, $R=R_{x}$ and the same maximum Hertzian contact pressure. In that case the dimensionless parameters characterising the equivalent line contact, i.e. $M_{1}$ and $L_{1}$, follow from the values of $M, L$ and $D$ for the elliptic contact:

$L_{1}=L$

and

$M_{1}=0.5(1.5 M \kappa)^{2 / 3}\left(\frac{(1+D) \pi}{4 E_{\mathrm{c}}}\right)^{1 / 3}$

Eq. (13) follows from substitution of $R=R_{x}$ and the equivalent line contact load per unit width $w$ obtained by equating the Hertzian pressures with $a=a_{x}$ :

$\left.\begin{array}{ll}\text { ne contact: } \quad p_{\bar{h}}=\frac{2 w}{\pi a} \\ \text { elliptical contact: } p_{\bar{h}}=\frac{3 F_{\kappa}}{2 \pi a_{x}^{2}}\end{array}\right\} w=\frac{3 F_{\kappa}}{4 a_{x}}$

and $a_{x}$ from Eq. (11) in the definition of the line contact dimensionless load parameter:

$M_{1}=\frac{w}{E^{\prime} R}\left(\frac{E^{\prime} R}{\eta_{\mathrm{o}} u_{\mathrm{s}}}\right)^{1 / 2}$ 